\title{
Giant Host Red Blood Cell Membrane Mimicking Polymersomes Bind Parasite Proteins and Malaria Parasites
}

\author{
Adrian Najer ${ }^{\text {sabc, Sagana Thamboo }}$, Cornelia G. Palivan, Hans-Peter Beck ${ }^{\mathrm{bc}}$, and Wolfgang Meier ${ }^{\mathrm{a}}$
}

§SCS-Metrohm Award for best oral presentation in Polymers, Colloids and Interfaces

\begin{abstract}
Malaria is an infectious disease that needs to be addressed using innovative approaches to counteract spread of drug resistance and to establish or optimize vaccination strategies. With our approach, we aim for a dual action with drug- and 'vaccine-like' activity against malaria. By inhibiting entry of malaria parasites into host red blood cells (RBCs) - using polymer vesicle-based (polymersome) nanomimics of RBC membranes - the life cycle of the parasite is interrupted and the exposed parasites are accessible to the host immune system. Here, we describe how host cell-sized RBC membrane mimics, formed with the same block copolymers as nanomimics, also bind the corresponding malaria parasite ligand and whole malaria parasites, similar to nanomimics. This was demonstrated using fluorescence imaging techniques and confirms the suitability of giant polymersomes (GUVs) as simple mimics for RBC membranes.
\end{abstract}

Keywords: Giant polymersomes · GUV $\cdot$ Malaria $\cdot$ Membrane mimics $\cdot$ Plasmodium

\section{Introduction}

Block copolymer-based vesicles, called polymersomes ${ }^{[1]}$ due to their increased mechanical stability compared to liposomes and broad chemical versatility to introduce e.g. stimuli-responsiveness - are considered ideal candidates for drug delivery purposes, as nanoreactors, as artificial organelles inside cells, or as simple cell mimics. ${ }^{[2,3]}$ Our recently developed nanomimic strategy against infectious diseases is based on such polymer vesicles imitating a host cell membrane at the nanoscale (nanomimics), which was achieved by mixing two distinct block copolymers: vesicle-forming poly(2-methyl-2-oxazoline)-blockpoly(dimethylsiloxane)-block-poly(2methyl-2-oxazoline) (PMOXA- $b$-PDMS$b$-PMOXA), and PDMS- $b$-heparin. ${ }^{[4,5]}$ Giant polymersomes (giant unilamellar vesicles, GUVs), several micrometers in diameter, are increasingly acknowledged as interesting model systems of very simplified cell mimics. ${ }^{[6]}$

\footnotetext{
${ }^{*}$ Correspondence: Prof. Dr. W. Meier ${ }^{\mathrm{a}}$

E-mail: wolfgang.meier@unibas.ch

aDepartment of Chemistry

University of Basel

Klingelbergstrasse 80

$\mathrm{CH}-4056$ Basel

bSwiss Tropical and Public Health Institute

Socinstrasse 57 Postfach

$\mathrm{CH}-4002$ Basel

University of Basel

Petersgraben 2, $\mathrm{CH}-4002$ Basel
}

Malaria, a disease caused by Plasmodium spp. parasites that are transmitted by Anopheles mosquitoes - killing more than 600'000 people each year ${ }^{[7]}-$ is our current target. When reaching the blood stage cycle, the malaria parasite form called merozoite attaches to and enters red blood cells (RBCs), where the parasite asexually divides to form more parasites, which egress from the infected RBC (iRBC) after about 48 hours and infect more RBCs. ${ }^{[8]}$ Heparan sulphate (very similar to heparin) on RBCs is considered as being responsible for the initial attachment of merozoite to the RBC by interaction with Plasmodium falciparum merozoite surface protein 1-42 $\left(\mathrm{PfMSP}_{42}\right){ }{ }^{[9]}$ With our approach we block the malaria blood stage life cycle using RBCmembrane-mimicking polymersomes, which block the parasite after egress from iRBCs, inhibit invasion, and expose the infective merozoites to the immune system. If successful in vivo, this artificial inhibition of merozoite invasion could lead to an immune boost against extracellular merozoites. ${ }^{[4]}$

Here, we demonstrate that RBC membrane mimics formed at a larger size, but with similar hollow sphere architecture (heparin GUVs), can also bind the parasite protein $P f \mathrm{MSP}_{42}$ and the whole Plasmodium parasite (merozoite form) in a similar fashion as their nano-sized counterparts, the nanomimics. ${ }^{[4,5]}$ This indicates that GUVs indeed represent a suitable model of small polymersomes, with the advantage of a size close to that of RBCs. Certain aspects, e.g. binding of $P f \mathrm{MSP}_{42}$ to polymersomes can be studied with GUVs using methods that would not be suitable for the nanostructure (e.g. confocal laser scanning microscopy (CLSM) to study protein binding).

\section{Experimental}

\section{Materials}

PDMS- $b$-heparin, PMOXA- $b$-PDMS$b$-PMOXA, and PfMSP1 $1_{42}-\mathrm{OG} 488$ synthesis and characterization can be found in our previous publications. ${ }^{[4,5]}$

\section{Giant Polymersome Formation}

Giant polymersome (GUV) formation was accomplished according to the standard electroformation technique ${ }^{[10]}$ using a Nanion Vesicle Prep Pro setup (Nanion Technologies, Munich, Germany). A freshly cleaned ITO-coated glass slide was first plasma-treated (Plasma Cleaner, PDC002, Harrick Plasma, Ithaca, New York, USA), and then, a thin polymer film was deposited on it. For control GUVs (only PMOXA- $b$-PDMS- $b$-PMOXA) a solution of PMOXA- $b$-PDMS- $b$-PMOXA in ethanol $(6 \mathrm{mg} / \mathrm{mL}, 40 \mu \mathrm{L})$ was dispersed on the ITO-coated side of the glass slide and ethanol was subsequently evaporated using a vacuum chamber for $30 \mathrm{~min}$ (Plasma Cleaner, PDC-002, Harrick Plasma, Ithaca, New York, USA). For the heparin GUVs a mixture of PMOXA- $b$-PDMS- 
$b$-PMOXA in ethanol $(6 \mathrm{mg} / \mathrm{mL}, 40 \mu \mathrm{L})$ and PDMS- $b$-heparin in ethanol $(4 \mathrm{mg} /$ $\mathrm{mL}, 10 \mu \mathrm{L}$ ) corresponding to a ratio of wt/ wt 85:15 was used. Afterwards, a greased O-ring was put around the polymer film on the glass slide to form a chamber and then an aqueous sucrose solution $(300 \mathrm{mM}$, $600 \mu \mathrm{L}$ ) was added to hydrate the polymer film. After covering by another ITO-coated glass slide, the sample was exposed to an ac current $(3.0 \mathrm{~V}, 3.0 \mathrm{~Hz})$ at room temperature. For control GUVs we performed electroformation overnight and for heparin GUVs electroformation was conducted for one hour. Finally, the GUV solutions were transferred to Eppendorf tubes and stored at $4{ }^{\circ} \mathrm{C}$.

\section{Fluorescence Microscopy}

Fluorescence imaging of protein interaction with giant polymersomes was performed on a confocal laser scanning microscope (Zeiss CLSM 510-META/ Confocor2, Carl Zeiss, Jena, Germany). A freshly plasma-cleaned 8-well microscopy chamber (Nunc Lab-Tek Chamber Slide System, Thermo Fisher Scientific) was filled with PBS (200 $\mu \mathrm{L}, \mathrm{pH} 7.2)$, GUVs $(20 \mu \mathrm{L}$ in $300 \mu \mathrm{mM}$ sucrose $)$, Bodipy630 (10 $\mu \mathrm{L}, 72 \mu \mathrm{M})$ in PBS, and PfMSP1 $_{42}-\mathrm{OG} 488$ in PBS $(5 \mu \mathrm{L}, 2.5 \mu \mathrm{M})$. For the CLSM measurements a water immersion objective (C-Apochromat, $40 \mathrm{x}$ magnification, NA of 1.2) was used. The OG488 signal was obtained by excitation with an Ar laser $(\lambda=488 \mathrm{~nm})$ with dichroic mirrors HFT (main beam splitter) 488 and NFT (secondary beam splitter) 490 with the band-pass BP 500-550 filter. HFT $488 / 543 / 633$, NFT 545 and the long-pass LP 650 filters were used to collect the fluorescence signal of Bodipy630. The pinhole size was set to $70 \mu \mathrm{m}$ for the green channel, and $90 \mu \mathrm{m}$ for the red channel, respectively. All settings (including laser power, detector gains) were kept constant for imaging of positive and negative samples.

Fluorescence imaging of heparin GUVs with merozoites was performed on a Leica DM 5000B fluorescence microscope using the appropriate filters.

\section{Study Interaction of Giants and Merozoites}

Plasmodium falciparum 3D7 parasite culturing and merozoite isolation was performed as described elsewhere. ${ }^{[4,11]}$ Briefly, a $30 \mathrm{~mL}$ petri dish filled with human RBCs at 5\% haematocrit and 5-6\% parasitemia (iRBC late stages) was used for merozoite isolation. First, late stage iRBCs were separated from RBCs and young iRBCs by Percoll density gradient. After maturation to schizont stage parasites, they were concentrated to $1 \mathrm{~mL}$ in malaria culture medium, and viable merozoites were mechanically released using $1.2 \mu \mathrm{m}$ syringe filters. They were immediately mixed with $300 \mu \mathrm{L}$ of a heparin GUV solution in $300 \mathrm{mM}$ sucrose, gently shaken, and incubated under static conditions at $37^{\circ} \mathrm{C}$ for $15 \mathrm{~min}$. Subsequently, $2 \mu \mathrm{L}$ of $1 \mathrm{mg} /$ mL Hoechst (DNA stain) was added and merozoite/giant mixture was fixed in $2 \%$ paraformaldehyde $/ 0.2 \%$ glutaraldehyde in $150 \mathrm{mM}$ phosphate buffer ( $\mathrm{pH} 7.4$ ) overnight. After gentle centrifugation (3500 $\mathrm{RCF}$ ) for $5 \mathrm{~min}, 10 \mu \mathrm{L}$ of the pellet was mounted on a microscopy slide.

\section{Results and Discussion}

Recently, we demonstrated that polymersome-based nanomimics of host RBC membranes efficiently block malaria parasite invasion into host RBCs in vitro; nanomimics were more than two orders of magnitude more potent invasion inhibitors compared to soluble receptor-like molecules (heparin), when $\mathrm{IC}_{50}$ values were compared based on the heparin concentration in the assays. ${ }^{[4,5]}$ Furthermore, we measured that several heparin chains on a single nanomimic bound parasite ligands PfMSP1 ${ }_{42}$-OG488 with a high affinity $\left(\mathrm{K}_{\mathrm{d}}\right.$ of $12.1 \pm 1.6 \mathrm{nM}$ ) using fluorescence crosscorrelation spectroscopy (FCCS) of green labelled ligand and red labelled nanomimics. ${ }^{[5]}$ This multiple binding with high affinity is the basis for a strong multivalent interaction of nanomimic and merozoite, explaining the potent invasion inhibition by nanomimics. ${ }^{[5]}$

Herein, host RBC membrane-mimick- ing polymersomes were formed at a larger size (micrometer scale, heparin GUVs) to study malaria protein and whole parasite interaction with RBC-sized polymersomebased mimics (Fig. 1). The same two block copolymers previously used for nanomimic formation were also used to form these giant mimics. PDMS $_{65}-b$-heparin was mixed with $\mathrm{PMOXA}_{5}-b$ - $\mathrm{PDMS}_{58}{ }^{-}$ $b$-PMOXA 5 (or PMOXA $_{9}-b$-PDMS ${ }_{67}-b-$ $\mathrm{PMOXA}_{9}$ ) using $15 \mathrm{wt} \%$ of the first block copolymer and subsequent electroformation, yielding heparin GUVs. GUVs made from $\mathrm{PMOXA}_{5}-b-\mathrm{PDMS}_{58}-b-\mathrm{PMOXA}_{5}$ without the heparin-functionalized copolymer were used as controls.

\section{Giant Host Red Blood Cell Membrane Mimicking Polymersomes Bind Plasmodium Proteins}

Formation of GUVs and heparin GUVs was successfully achieved in both cases using electroformation. Usually, a large number of mainly unilamellar, univesicular polymersomes were formed, whereas only few giants with multivesicular structures were found. Interestingly, these artificial and flexible membranes possess a thickness of about $11 \mathrm{~nm},{ }^{[4]}$ whilst all the membrane components can laterally diffuse within the membrane, ${ }^{[12,13]}$ but still form stable vesicles with several tens of micrometers in diameter. We performed CLSM to study the interaction of fluorescently labelled Plasmodium ligand PfMSP1 $1_{42}-\mathrm{OG} 488$ and heparin, the receptor-like molecule known to bind $P f \mathrm{MSP}_{42},{ }^{, 9]}$ on the surface

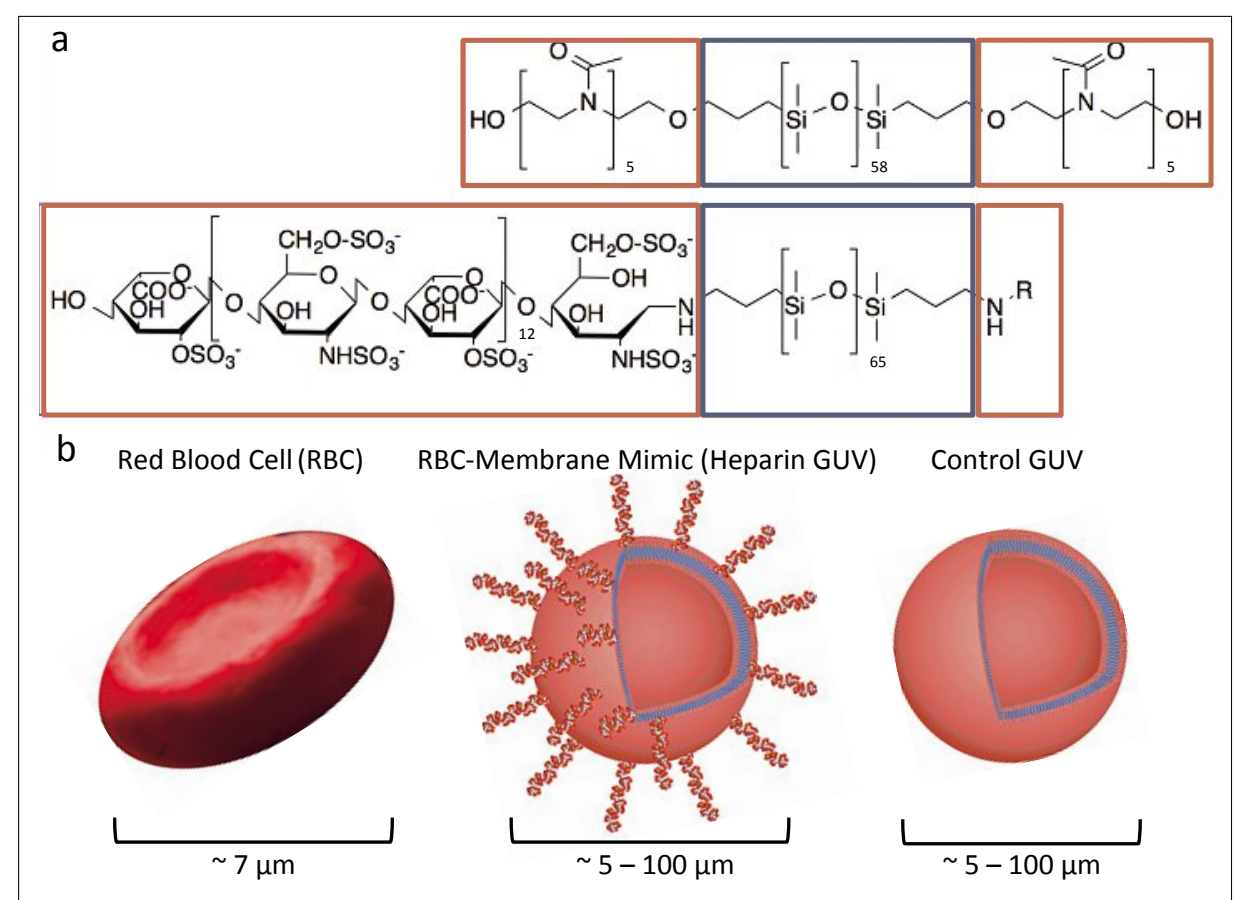

Fig. 1. a) Representation of the chemical structure of the two used block copolymers: $P_{M O X A_{5}-b-}$ $\mathrm{PDMS}_{58}-b-\mathrm{PMOXA}_{5}$ and $\mathrm{PDMS}_{65}-b$-heparin ${ }_{12}$. $\mathrm{R}$ is either another heparin chain or hydrogen. b) Schematic representation of a red blood cell (RBC), a RBC-membrane-mimetic giant polymersome (heparin GUV), and a control GUV without the heparin-containing copolymer. 
of heparin GUVs. This is only possible with GUVs and not with the nanomimics, because of resolution limitations. The membrane of control GUVs and heparin GUVs were labelled with the hydrophobic dye Bodipy630 for visualizing all the membranes in the red channel (Fig. 2). The hydrophobic dye spontaneously incorporates into the hydrophobic part of the membranes (PDMS).

The receptor-ligand interaction was followed after mixing control GUVs and heparin GUVs with green-labelled PfMSP1 ${ }_{42}$-OG488, respectively. In the control experiments, where GUVs without the receptor-like polysaccharide were used, no significant interaction with the parasite protein was observed as expected (Fig. 2c,d). Only the heparin GUVs, which mimic the host RBC membrane, were covered with PfMSP1 $1_{42}$-OG488 proteins on the surface, which appeared as green rings, whilst the exact same imaging settings as in the experiments with GUVs were used (Fig. 2a,b). Therefore, we could successfully observe the interaction of heparin on GUVs with the Plasmodium ligands PfMSP1 $1_{42}$ using conventional CLSM imaging. This confirms measurements performed using nanomimics and fluorescence-based diffusion measurements (fluorescence correlation spectroscopy (FCS), and FCCS) with the same PfMSP1 $1_{42}^{-}$ OG488 ligands of merozoites. ${ }^{[4,5]}$ This indicates that the membrane-bound receptorlike molecules (heparin) were also exposed on the large heparin GUVs after electroformation, similarly to the nano-scaled versions thereof (nanomimics). Therefore, GUVs are well suited to study certain biophysical aspects of the interaction between the parasite proteins and membrane mimics with a size relevant biologically, which support the conclusions already reported for the corresponding nanoversion system (nanomimics). ${ }^{[4,5]}$

\section{Giant Host Red Blood Cell \\ Membrane Mimicking Polymersomes Bind Plasmodium Merozoites \\ Successful binding of parasite proteins} to heparin GUVs encouraged us to perform experiments with whole Plasmodium merozoites to check for binding of viable parasites to heparin GUVs. This was studied using fluorescence microscopy after incubation of heparin GUVs with purified, viable Plasmodium falciparum merozoites, which is the most aggressive, life-threatening species of malaria parasites. The membrane of heparin GUVs was again visualized by red, hydrophobic dye (Bodipy630). Fluorescence images clearly demonstrate that merozoites also bind to RBC-sized host membrane-mimetic polymersomes (Fig. 3). In both examples shown, the

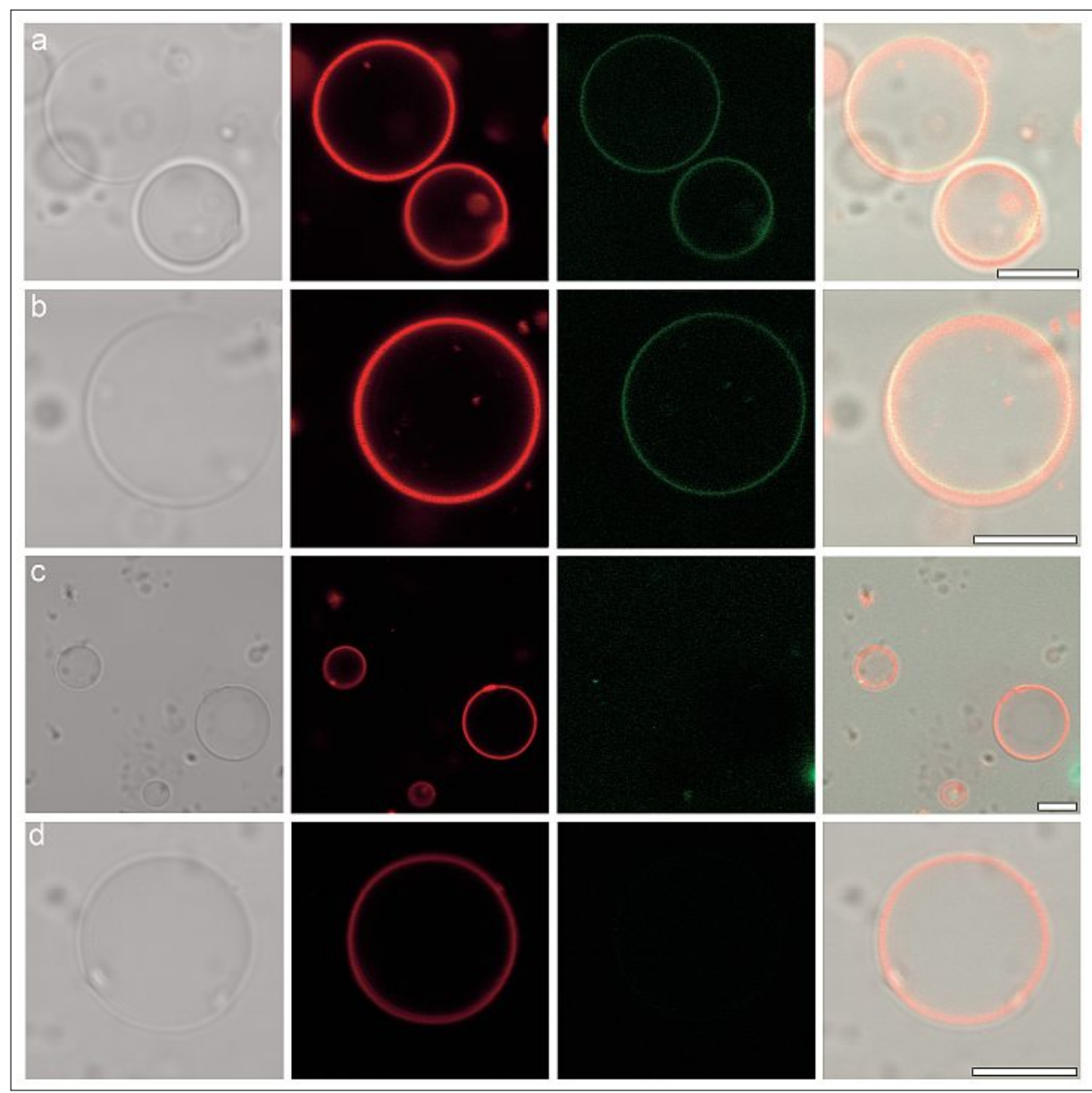

Fig. 2. CLSM images of heparin GUVs (a, b) and control GUVs (c, d), which were mixed with the Plasmodium parasite ligand $\mathrm{PfMSP}_{42}$-OG488 to investigate the ligand-receptor interaction; only when the receptor was presented on the surface (heparin GUVs), the parasite ligand bound to the GUVs, control GUVs showed no non-specific interaction with the protein. Left: DIC image. Middle left: Bodipy630 in the hydrophobic part of the GUV membranes. Middle right: OG488 on $\mathrm{PfMSP}_{42}$. Right: Merge. Scale bars: $10 \mu \mathrm{m}$. merozoites slightly indented the membrane of heparin GUVs upon binding. This deformation of the polymersome structure upon merozoite binding was also seen with nanomimics as previously demonstrated by electron microscopy of ultrathin slices of merozoite-nanomimic complexes. ${ }^{[4]}$

This confirms that large-scaled poly- mer vesicles can mimic - at a very primitive stage - the membrane of the host RBC to such an extent that binding of Plasmodium proteins and whole malaria parasites is achieved. The large size of GUVs allows the analysis of certain aspects by conventional imaging methods. Naturally, for a real in vivo application the nano-scaled

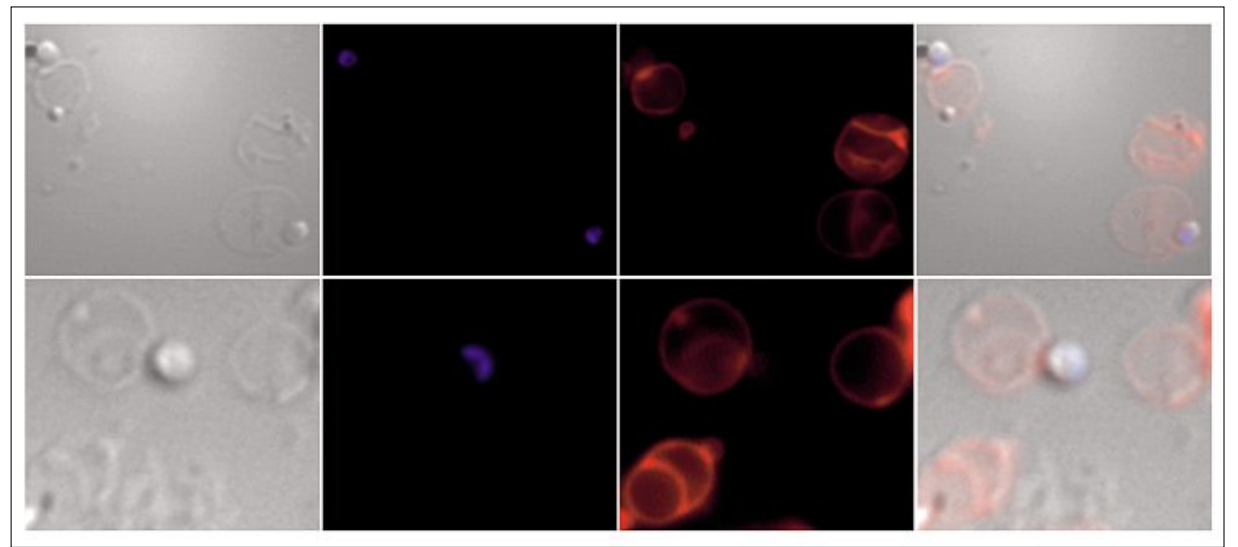

Fig. 3. Fluorescence microscopy images of giant host RBC-mimicking polymersomes (red) that bound Plasmodium merozoites (blue, DNA stain). Left: DIC image. Middle left: Hoechst, DNA stain. Middle right: Bodipy630, staining polymersome membranes. Right: Merge. Merozoites are about $1.5 \mu \mathrm{m}$ long. 
versions are preferable, but certain aspects are difficult to analyze using only nanosized structures.

\section{Conclusion}

Host RBC-membrane-mimicking polymersomes (heparin GUVs) were successfully formed at a corresponding RBC size (about $7 \mu \mathrm{m}$ disk diameter) using two block copolymers and electroformation technique. Only when the second copolymer, containing the receptor-like molecule (heparin), was present in the GUV membrane, the parasite ligand PfMSP1 bound to the GUVs. Furthermore, whole, viable, and RBC-infecting Plasmodium falciparum merozoites interacted and deformed these large-sized RBC membrane mimics (heparin GUVs). These are important steps towards understanding and de- fining this concept and to advance closer to a real biomedical application.

\section{Acknowledgements}

The Swiss National Science Foundation, NCCR 'Molecular Systems Engineering', and the University of Basel are acknowledged for financial support. A.N. gratefully acknowledges Metrohm Schweiz AG and the Swiss Chemical Society for the award. Authors thank S. Kasper for synthesizing PMOXA- $b$-PDMS- $b$-PMOXA and E. Angov (Division of Malaria Vaccine Development, Walter Reed Army Institute of Research, Silver Spring, MD, USA) is kindly acknowledged for providing $P f \mathrm{MSP}_{42}$.

\section{Received: December 27, 2015}

[1] B. M. Discher, Science 1999, 284, 1143.

[2] A. Feng, J. Yuan, Macromol. Rapid Commun. 2014, 35, 767.

[3] C. G. Palivan, R. Goers, A. Najer, X. Zhang, A. Car, W. Meier, Chem. Soc. Rev. 2016, 42, 377.
[4] A. Najer, D. Wu, A. Bieri, F. Brand, C. G. Palivan, H.-P. Beck, W. Meier, ACS Nano 2014 8,12560 .

[5] A. Najer, S. Thamboo, J. T. Duskey, C. G. Palivan, H.-P. Beck, W. Meier, Macromol. Rapid Commun. 2015, 36, 1923.

[6] M. Marguet, C. Bonduelle, S. Lecommandoux, Chem. Soc. Rev. 2012, 42, 512

[7] World Health Organization, 'World Health Statistics 2014', Geneva, 2014

[8] A. F. Cowman, D. Berry, J. Baum, J. Cell Biol. 2012, 198, 961.

[9] M. J. Boyle, J. S. Richards, P. R. Gilson, W. Chai, J. G. Beeson, Blood 2010, 115, 4559.

[10] M. I. Angelova, D. S. Dimitrov, Faraday Discuss. Chem. Soc. 1986, 81, 303.

[11] M. J. Boyle, D. W. Wilson, J. S. Richards, D. T. Riglar, K. K. Tetteh, D. J. Conway, S. A. Ralph, J. Baum, J. G. Beeson, Proc. Natl. Acad. Sci. USA 2010, 107, 14378.

[12] F. Itel, M. Chami, A. Najer, S. Lörcher, D. Wu, I. A. Dinu, W. Meier, Macromolecules 2014, 47, 7588.

[13] F. Itel, A. Najer, C. G. Palivan, W. Meier, Nano Lett. 2015, 15, 3871 . 